\section{Die eingeklemmte Autonomie}

\author{
R. Baumann-Hölzle, D. Eich-Höchli
}

Am 28. Sept. 2000 feierte das Drop-In mit einem Symposium an der Psychiatrischen Universitätsklinik in Zürich sein dreissigjähriges Bestehen. Die folgenden Ausführungen wurden an dieser Veranstaltung vorgetragen. Sie sind ein klärender, interdisziplinärer Beitrag zur gegenwärtigen Diskussion rund um die schweizerische Drogenpolitik.

In der heute gängigen psychiatrischen Diagnostik wird nicht von Sucht, sondern von Abhängigkeitsstörung gesprochen. Beim ethischen Diskurs über Abhängigkeitsstörungen steht die Frage nach der Verantwortung der Patientinnen und Patienten im Zentrum: Inwieweit kann ein Mensch für seine $\mathrm{Ab}$ hängigkeit von bestimmten Stoffen verantwortlich gemacht werden? Die Antwort auf diese Frage bestimmt die Handlungs- und Therapiekonzepte. Im folgenden ist eine typische Drogenkarriere beschrieben:

Lukas Meier (Name geändert), 20jährig, leidet seit dem 16. Lebensjahr an verschiedenen Abhängigkeitsstörungen: mit 16 Jahren begann er zu rauchen (Nikotin), sechs Monate später kam Haschisch (Cannabis) dazu. Mit 18 Jahren sniffte er Heroin (Opioid) und wechselte 19 jährig zum i.v. Konsum verbunden mit zunehmenden Mengen an Kokain. Die Entzugssymptome bekämpfte er mit Rohypnol (Beruhigungsmittel). Der soziale Abstieg war gekennzeichnet von Lehrabbruch bis hin zur Arbeitslosigkeit, Verlust des Freundeskreises und Stress in der Herkunftsfamilie und begann zweieinhalb Jahre, bevor der erste Kontakt mit institutionellen Helfern erfolgte und sich damit die Frage der angemessenen Behandlung stellte.

Das Konsumieren von illegalen Drogen, in unserem Zusammenhang Heroin, ist in der Schweiz verboten. Die Missachtung dieses Verbotes wird bestraft. Lange Zeit stand bei der politischen Auseinandersetzung mit diesem Thema vorwiegend die Repression der straffälligen Konsumierenden im Vordergrund und zwischen Konsum, Missbrauch und Abhängigkeitsstörung wurde nicht unterschieden.

Korrespondenz:

Dr. theol. Ruth Baumann-Hölzle

Dialog Ethik

Gloriastrasse 18

CH-8006 Zürich
Die Verantwortung für das Konsumieren wird hier impliziert. Werden hingegen gelegentlicher Konsum, missbräuchlicher Konsum und Abhängigkeitsstörung getrennt betrachtet, lassen sich verschiedene Verantwortlichkeiten formulieren.

\section{Freier Wille und Abhängigkeit}

Die menschliche Existenz zeichnet sich durch die Ambivalenz von Freiheit und Abhängigkeit aus. Ein integrales Menschenbild integriert die freien und die abhängigen Seiten in einer ganzheitlichen Sichtweise vom Menschen. Freiheit wird in diesem Kontext als die Freiheit zum bewussten Gestalten von existentiellen Abhängigkeiten verstanden. Eine Handlung, welche die menschlichen Abhängigkeiten unbeachtet lässt, ist "willkürlich" zu nennen.

Freier Wille und Leiblichkeit sind bei den Menschen interdependent, was Konsequenzen für ihre Verantwortlichkeit hat. Anhand des Begriffes der Sättigung kann die Interdependenz zwischen Leiblichkeit und Wille deutlich gemacht werden. Der freie Wille ist an die Leiblichkeit gebunden. Diese Leiblichkeit macht den Menschen physisch und psychisch $\mathrm{zu}$ einem bedürftigen Wesen, das gesättigt werden muss und will. Je gesättigter ein Mensch psychisch und physisch ist, desto freier kann er handeln. Erwachsene Menschen zeichnen sich denn auch gegenüber Kindern dadurch aus, dass sie ihre existentiellen Abhängigkeiten autonom gestalten können, indem sie für die Art und Weise, wie sie ihren existentiellen Hunger befriedigen wollen, einen bestimmten Freiraum, denjenigen ihrer persönlichen Willensfreiheit, besitzen. Zwischen dem freien Willen und der Abhängigkeit besteht bei einem gesunden Menschen ein Gleichgewicht.

Ungestillte existentielle Bedürfnisse können vom Menschen in einem gewissen Grad und mit verschiedenen Mitteln kompensiert werden, sei es mit Sport, Arbeit, Essen, Genussmitteln, usw. Diese Kompensationen, welche den ungestillten Hunger existentieller Abhängigkeit ausgleichen, stellen das Gleichgewicht zwischen Freiheit und Abhängigkeit wieder her. Sie ermöglichen dem Menschen, sein Leben frei zu gestalten.

\section{Abhängigkeitsstörungen}

Solche Kompensationen, welche ungestillte existentielle Bedürfnisse abdecken, können nun ihrerseits zum Selbstzweck werden, indem sie nicht mehr Abhängigkeiten auf Freiheit hin kompensieren, sondern selber neue Abhängigkeiten erzeugen. Das Gleichgewicht zwischen Autonomie und Abhängigkeit geht dadurch verloren und es kommt zu Abhängigkeitsstörungen. Der abhängigkeitsgestörte Patient zeichnet sich durch einen kaum stillbaren Hunger nach einer Substanz, nach Arbeit, nach sportlicher Aktivität, nach einem Menschen, usw. aus. Er verliert seine Entscheidungsfreiheit gegenüber einem Kom- 
pensationsmechanismus, es kommt zu physischen und/oder psychischen Schädigungen und er wird zum Abhängigkeitskranken. Abhängigkeitsstörungen treten in verschiedenen Graden und meist auch als Mehrfachhunger auf. Erst wenn der Hunger, der der Abhängigkeitsstörung zugrunde liegt, gestillt ist, sind freie Entscheidungen bei einem abhängigkeitsgestörten Menschen wieder möglich.

Aus dem Gesagten kann die Schlussfolgerung gezogen werden, dass durch die Interdependenz von Leiblichkeit und Wille die Abhängigkeitsstörung nicht nur die Leiblichkeit, sondern auch den Willen eines Menschen beeinflussen kann. So ist bekannt, dass Diabetes Depressionen erzeugen kann und bestimmte chemische Stoffe Willensveränderungen bewirken. Die Autonomie eines Menschen kann so eingeklemmt sein zwischen verschiedenen Formen abhängigkeitsgestörter Leiblichkeit und abhängigkeitsgestörtem Willen. Die eingeklemmte Autonomie macht die medizin-ethische Entscheidungsfindung oft schwierig, denn in dieser Situation fällt es schwer, den freien Willen eines Menschen zu erkennen. Ist eine Therapieverweigerung, zu der jeder Patient das Recht hat, Ausdruck einer Depression oder des freien Willens?

\section{Abhängigkeitsstörungen und Genetik}

Nach neusten Erkenntnissen haben Abhängigkeitsstörungen auch eine genetische Komponente. Geht man von einem Menschenbild aus, wonach der Mensch allein durch seine Gene determiniert wird, so besitzt ein Mensch gegenüber seiner genetischen Bestimmung keinen freien Willen. Die Gene werden so für den einzelnen Menschen zum persönlichen Schicksal. Wird dieses Menschenbild handlungsrelevant, so kann ein abhängiger Mensch in keiner Art und Weise für sein Verhalten zur Verantwortung gezogen werden. Geht man andererseits von einem völlig freien Willen aus, wonach sich ein Mensch gegenüber seinen Genen absolut frei verhalten kann, so wird die "Abhängigkeitsstörung" zur persönlichen Verantwortung. Weder die eine noch die andere Vorstellung vom Menschen sind dem Sein des Menschen angemessen.

Werden Menschen ohne genetische Disposition für Abhängigkeitsstörungen psychisch und physisch ausreichend gesättigt, so ist die Chance klein, dass sie eines Tages abhängig werden. Werden hingegen Menschen ohne genetische Disposition physisch und psychisch nicht ausreichend gesättigt, so entsteht durch diesen Mangel an Sättigung ein gewisses Abhängigkeitspotential, indem sie mit einem oder mehreren Stoffen ihren physischen und psychischen Hunger zu stillen hoffen. Demgegenüber kann bei Menschen mit einer genetischen Abhängigkeitsdisposition der latent vorhandene Hang zur gestörten Abhängigkeit durch eine optimale psychische und physische Sättigung kompensiert werden. Umgekehrt führt bei diesen Menschen psychischer Hunger vermehrt zu Abhängigkeitsstörungen.

\section{Ethische Konsequenzen}

Leiblichkeit und Wille bilden beim Menschen eine Einheit. Physische und psychische Abhängigkeitsstörungen sind deshalb moralisch in gleicher Art und Weise $\mathrm{zu}$ bewerten. Es gibt keine rationalen Gründe, Menschen mit einer Abhängigkeitsstörung von sogenannt «illegalen" Stoffen moralisch anders zu bewerten als Menschen, die von legalen Stoffen abhängigkeitsgestört sind. Krankheiten sind immer Formen von Abhängigkeitsstörungen. Damit ein Mensch seine Freiheit trotzdem wahrnehmen, d.h. seine Abhängigkeiten bewusst gestalten kann, ist er oder sie auf eine künstliche Sättigung von aussen angewiesen, wie dies bei einer chronischen Diabetes bei der Abhängigkeit vom Insulin oder beim Heroinabhängigen vom Methadon der Fall ist. Es ist nicht einzusehen, warum die Behandlung mit Insulin moralisch anders zu beurteilen ist als diejenige mit Methadon. Beide sollen und dürfen nur dann eingesetzt werden, wenn mit ihnen Schaden vermieden werden kann und Menschen ermöglicht wird, ihr Gleichgewicht zwischen Autonomie und Abhängigkeit wieder zu erreichen. Krankheit darf nicht zur Schuld werden.

\section{Handlungskonsequenzen}

Menschen mit einer Abhängigkeitsstörung von irgendeinem Stoff oder Verhalten wie Arbeit, Insulin oder Heroin gleich zu bewerten, bedeutet nicht, bei den abhängigkeitserzeugenden Substanzen keinen Unterschied zu machen und in Zukunft Heroin am Kiosk um die Ecke anzubieten. Dem unterschiedlichen abhängigkeitserzeugenden Potential und der kulturellen Einbettung eines Genussmittels einerseits und der gestuften Verantwortlichkeit andererseits ist bei der Ausgestaltung von Handlungsoptionen Rechnung zu tragen.

Grundsätzlich gilt, dass Gleiches mit Gleichem abgewogen werden soll. Stoffe, welche bei den meisten Menschen eine stark abhängigkeitserzeugende Wirkung haben, sollen auch in Zukunft verboten sein und sowohl deren Verkauf wie auch deren Konsum soll bei nicht abhängigkeitsgestörten Menschen als illegal gelten und bestraft werden. Hierunter fallen unter anderem Heroin und Kokain. Entsprechende Massnahmen sind gegen deren Handel und Konsum vorzunehmen. Nikotin hat ein ähnliches Abhängigkeitspotential wie Heroin. Nur gerade aus pragmatischen Gründen, weil sich ein Verbot von Nikotin heute nicht mehr durchsetzen liesse, ist Nikotin nicht als illegal zu erklären. Die Zugänglichkeit zum Nikotin ist aber sehr zu erschweren. Eine massive Verteuerung und ein Werbeverbot sind vordringlich, denn der Schutz von Menschen hat vor kommerziellen Interessen Vorrang.

Alkohol und Cannabis sind als Genussmittel ähnlich abhängigkeitserzeugend, doch in weit geringerem Ausmass als die Stoffe Heroin, Nikotin und Kokain. Ihre Gleichbehandlung drängt sich auf und 
die soeben beschlossene Liberalisierung des Cannabiskonsums ist insofern konsequent. Aber auch ihr Genuss ist nicht zu fördern. In diesem Zusammenhang ist ganz besonders auf den Jugendschutz hinzuweisen, denn Jugendliche sind in der Phase der Pubertätsentwicklung besonders gefährdet für Abhängigkeitsstörungen. So braucht es für einen Jugendlichen nur wenige Wochen täglichen Alkoholkonsums, um vom Alkohol abhängigkeitsgestört zu werden. Angesichts der Tatsache, dass Cannabis vorwiegend von Jugendlichen in der für Abhängigkeitsstörungen anfälligen Phase konsumiert wird, taucht trotzdem die Frage der Aufrechterhaltung des Cannabisverbotes auf. Es ist erwiesen, dass der Konsum von einem Genussmittel sofort ansteigt, sobald es nicht mehr als «illegal» behandelt wird.

Sobald bei einem Menschen aber eine Abhängigkeitsstörung von einem bestimmten Stoff, auch einem illegalen, vorliegt und dieser Konsum zur Selbst- oder Fremdgefährdung führt, ist ein Mensch krank. Wie alle anderen Patientinnen und Patienten hat er ein Anrecht auf Therapie. Genauso wie es der Zufall will, dass ein Mensch bei der Berührung mit einem Virus erkranken kann und dabei von einem bestimmten Medikament abhängig wird, will es der Zufall, dass ein Mensch bei der Berührung mit illegalen Drogen von einem bestimmten Stoff abhängig werden kann.
Liegt bei Menschen eine Abhängigkeitsstörung von Heroin vor, so soll diesen Menschen Methadon oder Heroin als Arzneimittel abgegeben werden können. Eine angemessene Behandlung beinhaltet also auch die Möglichkeit einer Methadon- oder Heroinbehandlung und zielt darauf hin, einem Menschen zu einem selbständigen Leben zu verhelfen. Die Drogenpolitik des Bundes mit ihren vier Säulen Prävention, Therapie, Schadensverminderung/Überlebenshilfe und Repression geht auf dieses Verständnis einer gestuften Verantwortlichkeit zurück.

\section{Solidargemeinschaft}

Die Angst der Menschen vor der eigenen Abhängigkeit führt dazu, abhängigkeitsgestörte Menschen nicht wahrzunehmen, abzulehnen und zu verurteilen. Die beste Prävention für Abhängigkeitsstörungen ist eine Gesellschaft, in deren Rahmen die Menschen ihren physischen und psychischen Hunger stillen können. Eine humane Gesellschaft ist verpflichtet, Schädigungen zu vermeiden. Menschen, deren Abhängigkeitsstörung zu Schädigungen führt und die nicht selber in der Lage sind, diesen Schaden zu verhindern und ihren Hunger selbst zu stillen, werden von einer humanen Gesellschaft ernährt und solidarisch getragen, damit sie ein möglichst selbständiges Leben führen können. Und hierzu können Methadon und in Ausnahmesituationen Heroin die geeigneten Arzneimittel sein. 